\title{
Estrategias para Acercar la Tecnología de Identificación por Radiofrecuencia a la Formación de Futuros Ingenieros Industriales
}

\author{
Alejandro Alvarez-Marin y Mauricio Castillo-Vergara \\ Universidad de La Serena, Departamento de Ingeniería Industrial, Benavente 980, La Serena-Chile \\ (e-mail: aalvarez@userena.cl, mhcastillo@userena.cl)
}

Recibido Jul. 20, 2014; Aceptado Sep. 29, 2014; Versión final recibida Nov. 5, 2014

\begin{abstract}
Resumen
Este estudio tiene como objetivo presentar la tecnología de identificación por radio frecuencia y determinar estrategias que sirvan para incorporarla a la formación de los futuros ingenieros industriales. Se analizó el funcionamiento y orígenes de la tecnología y se realizo una revisión de la literatura existente sobre sus aplicaciones. Luego se identificó su relación con áreas de conocimiento incluidas en el programa de Ingeniería Industrial (ciencias básicas, ciencias de la ingeniería, asignaturas de especialidad) y se analizaron las estrategias de adopción de la tecnología en las distintas actividades que desarrollan los estudiantes de Ingeniería Industrial en su formación profesional. Se concluye que las instituciones de educación superior tienen una gran oportunidad de potenciar su línea de acción al incorporar esta tecnología en la resolución de problemas de procesos empresariales y organizacionales, considerando su directa relación con las necesidades de la sociedad actual.
\end{abstract}

\section{Strategies to Bring Radio Frequency Identification Technology to the Training of Future Industrial Engineers}

\begin{abstract}
This study aims to understand the technology of radio frequency identification and to determine strategies that could serve to incorporate it to the formation of future industrial engineers. The functioning and origins of the technology also performed an applications presented in the literature were reviewed. Its relation to areas of knowledge included in the Industrial Engineering Curriculum was identified (basic sciences, engineering sciences, specialty subjects) and strategies for technology adoption in the various activities performed by the students of Industrial Engineering were analyzed. It is concluded that higher education institutions have a great opportunity to enhance its line of action by incorporating this technology in the solving business problems and organizational processes, considering its direct relation to the need of the present society.
\end{abstract}

Keywords: radio frequency, industrial engineering, higher education, professional formation 


\section{INTRODUCCIÓN}

La identificación por radiofrecuencia (RFID, por sus siglas en inglés) es un área de la identificación automática que está ganando impulso y es considerada por algunos como una de las tecnologías informáticas más penetrantes de la historia (Roberts, 2006). Esto se debe principalmente a su gran presencia en diversas industrias con numerosas aplicaciones prácticas que permiten optimizar o hacer más eficiente los procesos.

En los últimos años, su uso ha aumentado de manera exponencial. Es tal el impacto, que se predice que para el año 2016, habrá 450 veces más etiquetas RFID en uso que en 2010 (Aliaga et al., 2011). Aún más, de acuerdo a la última investigación del mercado de RFID desarrollada por IDTechEx en el año 2013, para dicho año se pronosticó una venta de 5.9 billones de etiquetas superando los 4.8 billones vendidos en 2012 (IDTechEx, 2013). Su enorme desarrollo se puede explicar en parte por el impulso que originó la política de uso obligatorio de etiquetas RFID adoptada por Wal-Mart, el Departamento de Defensa de los Estados Unidos, y compañías europeas tales como Metro y Tesco, lo que ha llevado a muchas organizaciones a estudiar detenidamente en qué puede ayudarles RFID y a qué aspectos deberían dar mayor importancia al momento de adoptar esta tecnología (Ngai et al., 2008a). Por otro lado, también se argumenta que el rápido aumento en el uso se debe a la caída de los costos de los dispositivos RFID (Knospe, 2004).

En el ámbito académico, existe un aumento significativo en la cantidad de publicaciones sobre esta tecnología. De hecho, muchas revistas, incluyendo Production and Operations Management y la International Journal of Production Economics, entre otras, poseen secciones especiales sobre el tema (Zhu et al., 2012). Así, la investigación RFID ha dado origen a un nuevo campo de la investigación académica basado en el trabajo previo sobre una gran variedad de disciplinas, tales como ingeniería electrónica, sistemas de información, ciencias de la computación, y estrategias de negocios (Ngai et al., 2008a). Como prueba del aumento notorio en el número de publicaciones RFID, la Figura 1 muestra la distribución de publicaciones por año, para el periodo 2004-2011.

El uso comercial de RFID, que comenzó hace más de 20 años, dio lugar a un cambio radical en la forma en que las organizaciones realizan el seguimiento de los bienes a través de la cadena de suministro (Roh et al., 2009). Sin embargo, sus aplicaciones no se limitan a eso; el rango potencial de uso va desde lo aparentemente trivial, tal como el control de inventario, hasta aplicaciones más futuristas, como electrodomésticos que administran la compra de alimentos, o ropa que es lavada de acuerdo a las instrucciones leídas automáticamente desde su etiqueta (Robshaw, 2006).

En vista de que las aplicaciones de la tecnología RFID atraviesan las más diversas áreas del conocimiento en ingeniería, es necesario que los futuros egresados de carreras relacionadas se familiaricen con dichas aplicaciones y que puedan asociarlas con el saber adquirido durante los años de formación académica. Para este fin, el presente artículo busca establecer estrategias para acercar la tecnología de identificación por radiofrecuencia a la formación de futuros Ingenieros Industriales, para lo cual se determinó realizar una revisión de la literatura existente, con el propósito de dar a conocer el estado de las investigaciones acerca de la tecnología RFID, proporcionando así información a los profesionales e investigadores de RFID sobre algunas aplicaciones importantes, detallando casos de estudio, orientando los futuros desafíos de RFID y determinar así las estrategias propuestas.

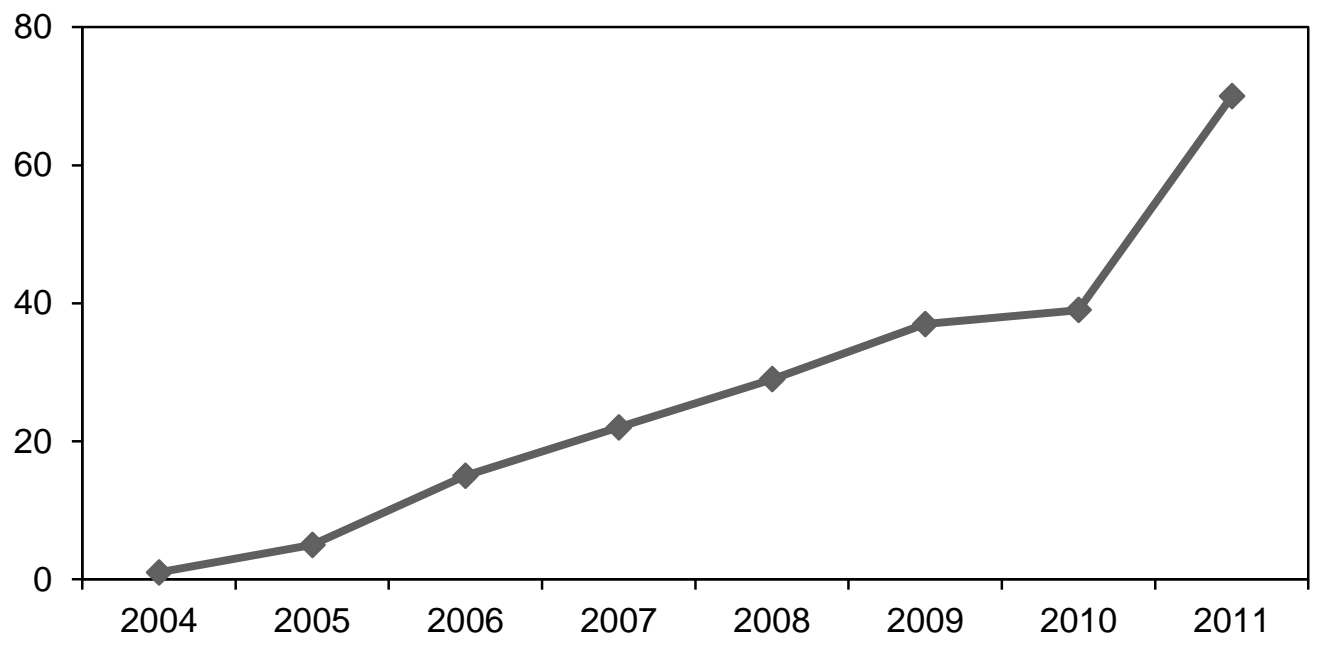

Fig. 1: Número de artículos por año para período 2004-2011 


\section{REVISION DE LA LITERATURA}

Se consideraron publicaciones desde el año 2006 hasta el año 2012. Para la búsqueda en la base de datos (Sciencedirect), se utilizó la siguiente combinación de términos: tecnología de identificación por radiofrecuencia, RFID e impacto de la tecnología RFID. Esta búsqueda resultó en alrededor de 1000 artículos únicos, los cuales fueron evaluados en relevancia por su título. De acuerdo con este, 269 artículos centrados en las aplicaciones RFID fueron seleccionados para realizar la revisión de sus resúmenes, los cuales dieron lugar a una selección de 218 artículos a ser leídos en su totalidad y clasificados de acuerdo a su ámbito de aplicación y estudio. La Tabla 1 resume la literatura considerada, indicando las aplicaciones con las cuales están relacionadas.

Tabla 1: Resumen la literatura considerada en el estudio

\begin{tabular}{|c|c|}
\hline Aplicación & Autores \\
\hline Ganadería & Reiners et al., 2009; Stankovski et al., 2012 \\
\hline Construcción & $\begin{array}{l}\text { Costin et al., 2012; Majrouhi Sardroud et al., 2012; Tzeng et al., 2008; Lu et al., } \\
\text { 2011; Wang, 2008; Chae y Yoshida, 2010; Lu et al., } 2011\end{array}$ \\
\hline Alimentos & $\begin{array}{l}\text { Jedermann et al., 2009; Ruiz-Garcia y Lunadei, 2011; Hong et al., 2011; } \\
\text { Ampatzidis y Vougioukas, 2009; Zhang y Li, 2012; Abad et al., 2009; Laniel y } \\
\text { Émond, 2010; Tan y Chang, 2010; Ngai et al., 2008b }\end{array}$ \\
\hline Salud & $\begin{array}{l}\text { Oztekin et al., 2010; Çakıcı et al., 2011; Qu et al., 2011; Bendavid y Boeck, } \\
\text { 2011; Najera et al., 2011; Parlak et al., 2012; Peris-Lopez et al., 2011; } \\
\text { Hohberger et al., 2012; Reyes et al., 2012; Kranzfelder et al., 2012; Fisher y } \\
\text { Monahan, } 2008\end{array}$ \\
\hline Bibliotecas & Xianyan y Jiayuan, 2011 \\
\hline $\begin{array}{l}\text { Logística y gestión de la cadena } \\
\text { de suministro }\end{array}$ & $\begin{array}{l}\text { Sarac et al., 2010; Tajima, 2007; Véronneau y Roy, 2009; Lee et al., 2011; } \\
\text { Björk et al., 2011; Bottani et al., 2010; Ko et al., 2011; Kapoor et al., 2011; Kim } \\
\text { y Garrison, 2010; Nativi y Lee, } 2012\end{array}$ \\
\hline Manufactura & Ngai et al., 2012; Wang et al., 2010; Trappey et al., 2009; Makris et al., 2012 \\
\hline Minería & Mishra et al., 2012 \\
\hline Retail & Condea et al., 2012; Rekik et al., 2008; Müller-Seitz et al., 2009 \\
\hline Servicios postales y de entrega & Zhang et al., 2006 \\
\hline Control de tráfico automovilístico & Wen, 2010 \\
\hline Turismo & Öztayşi et al., 2009 \\
\hline Educación & Akpınar y Kaptan, 2010 \\
\hline
\end{tabular}

\section{RESULTADOS}

A continuación se mostrarán los resultados obtenidos, identificando en primer lugar la tecnología RFID y sus orígenes, individualizando luego sus aplicaciones en el ámbito empresarial, finalizando con el análisis de las áreas del conocimiento en ingeniería relacionadas con esta tecnología.

\section{La tecnología RFID y sus orígenes}

La tecnología de identificación por radiofrecuencia no es nueva. Durante la segunda guerra mundial se utilizó para distinguir entre aviones del mismo bando que regresaban, y las del bando enemigo. Dicha tecnología se denominó Identidad Amigo o Enemigo (IFF, por sus siglas en inglés) y es considerada como la primera aplicación de la tecnología RFID (Domdouzis et al., 2007). La primera publicación sobre RFID tuvo lugar el año 1948, sin embargo sólo recientemente el tema ha llegado a ser de conocimiento público, llamando la atención de numerosas compañías debido a factores como la necesidad de aumentar la eficiencia y seguridad en la cadena de suministro, mejoras tecnológicas, e iniciativas de estandarización, entre otros (Leimeister et al., 2009). Aunque el concepto por sí mismo no es nuevo, si lo son sus características en cuanto a costos y tamaño que han logrado las tecnologías que proveen la funcionalidad RFID en los últimos años (Robshaw, 2006).

Respecto a su funcionamiento, un sistema RFID consiste en una pequeña etiqueta constituida por un chip de circuito integrado y una antena, que tiene la habilidad de responder a ondas de radio transmitidas desde el lector RFID con el propósito de enviar, procesar, y almacenar información. Dentro del sistema, se 
distinguen tres componentes básicos: la etiqueta (tag), el lector (reader), y el equipo que gestiona el procesamiento de datos. La etiqueta contiene información única del objeto al cual es adherida; el lector emite y recibe ondas de radio para leer la información almacenada en la etiqueta, y el equipo de procesamiento de datos procesa toda la información recolectada. Este equipo puede ser tan simple como un computador personal o tan complejo como el sistema de información administrativa completo de una empresa (Wu et al., 2006).

Como ya se mencionó, la etiqueta consta de un circuito integrado y una antena, la que define el rango de lectura de la etiqueta. Estas etiquetas se pueden dividir en dos categorías principales: etiquetas de sólo lectura, y etiquetas de lectura/escritura (Domdouzis et al., 2007). Por otra parte, el mismo autor también indica que pueden ser clasificadas en etiquetas pasivas y etiquetas activas. La diferencia radica en que las etiquetas pasivas dependen del campo electromagnético generado por el lector RFID, en cambio las activas tienen baterías incorporadas, lo que incrementa el rango de lectura debido a su no dependencia de dicho campo electromagnético. La Tabla 2 muestra las principales diferencias entre etiquetas pasivas y activas.

Las frecuencias de onda a las que puede operar un sistema RFID son variadas. La Tabla 3 muestra la norma ISO asociada a cada rango de frecuencia (Pirrone y Huerta, 2011), y las principales características de dicho rango (Domdouzis et al., 2007).

Tabla 2: Principales diferencias entre etiquetas pasivas y activas (Domdouzis et. al, 2007)

\begin{tabular}{|l|l|l|}
\hline & Etiquetas Activas & Etiquetas Pasivas \\
\hline Fuente de energía de la etiqueta & Interna a la etiqueta & Energía transferida desde el lector \\
\hline Disponibilidad de energía de la etiqueta & Continua & $\begin{array}{l}\text { Solo cuando se encuentra en el } \\
\text { campo del lector }\end{array}$ \\
\hline $\begin{array}{l}\text { Potencia de la señal requerida desde la } \\
\text { etiqueta al lector }\end{array}$ & Baja & Alta \\
\hline $\begin{array}{l}\text { Disponibilidad de potencia de señal } \\
\text { desde la etiqueta al lector }\end{array}$ & Alta & Baja \\
\hline Rango de comunicación & Amplio & Corto \\
\hline Colección de datos multi-etiqueta & $\begin{array}{l}\text { Escaneo de miles de etiquetas a } \\
\text { partir de una simple lectura } \\
\text { Escaneo sobre 20 etiquetas } \\
\text { moviéndose a más de 100 } \\
\text { millas/hora }\end{array}$ & $\begin{array}{l}\text { Escaneo de un centenar de } \\
\text { etiquetas cercanas a 3 metros a } \\
\text { partir de una simple lectura } \\
\text { Escaneo de 20 etiquetas } \\
\text { moviéndose a 3 millas/hora o más } \\
\text { lento }\end{array}$ \\
\hline Capacidad de detección & Habilidad para monitoreo continuo & $\begin{array}{l}\text { Se puede monitorear cuando la } \\
\text { etiqueta es alimentada por el lector }\end{array}$ \\
\hline $\begin{array}{l}\text { Capacidad de almacenamiento de } \\
\text { información }\end{array}$ & Amplia & Pequeña \\
\hline
\end{tabular}

Tabla 3: Norma ISO asociada a cada rango de frecuencia y principales características (Pirrone y Huerta, 2011; Domdouzis et. al., 2007)

\begin{tabular}{|l|l|l|l|l|l|}
\hline $\begin{array}{l}\text { Rango de } \\
\text { frecuencia }\end{array}$ & Norma Vigente & $\begin{array}{l}\text { Rango de } \\
\text { distancia }\end{array}$ & $\begin{array}{l}\text { Velocidad de } \\
\text { transferencia de } \\
\text { datos }\end{array}$ & $\begin{array}{l}\text { Penetración de las ondas } \\
\text { electromagnéticas }\end{array}$ & Usos \\
\hline Bajo $135 \mathrm{KHz}$ & $\begin{array}{l}\text { ISO/IEC } \\
18000-2: 2009\end{array}$ & $\begin{array}{l}\text { Menos de } \\
0.5 \mathrm{~m}\end{array}$ & $1 \mathrm{kbits} / \mathrm{s}$ & Agua pero no metal & $\begin{array}{l}\text { Identificación } \\
\text { animal }\end{array}$ \\
\hline $13,56 \mathrm{MHz}$ & $\begin{array}{l}\text { ISO/IEC } \\
18000-3: 2010\end{array}$ & $\begin{array}{l}\text { Sobre } 1.5 \\
\mathrm{~m}\end{array}$ & $25 \mathrm{kbits} / \mathrm{s}$ & Agua pero no metal & $\begin{array}{l}\text { Acceso y } \\
\text { seguridad }\end{array}$ \\
\hline $433 \mathrm{MHz}$ & $\begin{array}{l}\text { ISO/IEC } \\
18000-7: 2009\end{array}$ & $\begin{array}{l}\text { Sobre } 100 \\
\mathrm{~m}\end{array}$ & $100 \mathrm{kbits} / \mathrm{s}$ & Ni agua ni metal & Logística \\
\hline $860-960 \mathrm{MHz}$ & $\begin{array}{l}\text { ISO/IEC } \\
18000-6: 2013\end{array}$ & $\begin{array}{l}\text { De } 0.5 \text { a } 5 \\
\mathrm{~m}\end{array}$ & $100 \mathrm{kbits} / \mathrm{s}$ & Ni agua ni metal & Logística \\
\hline $2,45 \mathrm{GHz}$ & $\begin{array}{l}\text { ISO/IEC } \\
18000-4 .: 2008\end{array}$ & $10 \mathrm{~m}$ & Más de $100 \mathrm{kbits} / \mathrm{s}$ & Ni agua ni metal & $\begin{array}{l}\text { Pago de peaje } \\
\text { en carretera }\end{array}$ \\
\hline
\end{tabular}




\section{Aplicaciones}

Investigaciones realizadas por Liao et al. (2011) y Ngai et al. (2008a), entre otras, muestran importantes esfuerzos por clasificar la literatura existente. Ambos trabajos coinciden en dividir la investigación en 4 grandes categorías: Tecnología RFID, Aplicaciones RFID, Política y Seguridad, y Otros. Luego, cada categoría se divide en múltiples subcategorías. Esto nos ha servido de base para seleccionar un gran número de aplicaciones importantes que ofrece RFID para la ingeniería industrial, las que se detallan a continuación:

Ganadería: En el área de la ganadería, la tecnología RFID ha sido utilizada principalmente para registrar y rastrear animales, identificar animales expuestos a enfermedades, o controlar y erradicar amenazas veterinarias. La literatura muestra ejemplos sobre identificación de cerditos destetados (Reiners et al., 2009) y monitoreo de vacas lecheras (Stankovski et al., 2012).

Construcción: Las aplicaciones de RFID en construcción abarcan básicamente la identificación y monitoreo de materiales, trabajadores y equipos en tiempo real (Costin et al., 2012; Majrouhi Sardroud et al., 2012; Tzeng et al., 2008; Lu et al., 2011), así como la mejora de la gestión de la calidad (Wang, 2008), la prevención de accidentes (Chae y Yoshida, 2010) y por último el manejo de residuos de esta forma se evita la aparición de vertederos ilegales (Lu et al., 2011).

Alimentos: En lo que respecta a la industria alimenticia, se han identificado dos macro aplicaciones: primero, la relativa al registro, control y supervisión de los alimentos y de su estado en la cadena de suministro, como cuando se implementan sensores en etiquetas RFID que hacen posible el monitoreo de alimentos perecibles (Jedermann et al., 2009; Ruiz-Garcia y Lunadei, 2011; Hong et al., 2011; Ampatzidis y Vougioukas, 2009; Zhang y Li, 2012; Abad et al., 2009; Laniel y Émond, 2010), y en segundo término, la concerniente a la mejora en la gestión de restaurantes y empresas afines (Tan y Chang, 2010; Ngai et al., 2008b).

Salud: El sector salud presenta uno de los usos más amplios de la tecnología RFID. Las aplicaciones abarcan la administración de activos médicos e inventario farmacéutico (Oztekin et al., 2010; Çakıcı et al., 2011; Qu et al., 2011; Bendavid y Boeck, 2011), el monitoreo de pacientes, su salud y sus respectivos medicamentos (Najera et al., 2011; Parlak et al., 2012; Peris-Lopez et al., 2011), el monitoreo preciso de la corruptibilidad de la sangre para transfusión en los bancos de sangre (Hohberger et al., 2012), y la identificación de instrumentos que en estado no apto para intervención médica (Reyes et al., 2012; Kranzfelder et al., 2012). Además, se ha analizado la dimensión social del uso de la tecnología RFID en hospitales (Fisher y Monahan, 2008).

Bibliotecas: La tecnología RFID puede cambiar la manera en la que se lleva a cabo la gestión de las bibliotecas al mejorar la circulación de libros, prevención de robos o ayudar a detectar daños en libros (Xianyan y Jiayuan, 2011).

Logística y gestión de la cadena de suministro: La logística es otra de las áreas con mayor aplicación de la tecnología RFID. En este caso, esta tecnología permite mejorar los beneficios potenciales de la gestión de la cadena de suministro mediante la mejora en la trazabilidad de los objetos, mejora en la precisión de la información y en la respuesta ante cambios inesperados en la oferta y la demanda, y la reducción del efecto látigo (Sarac et al., 2010; Tajima, 2007; Véronneau y Roy, 2009; Lee et al., 2011; Björk et al., 2011; Bottani et al., 2010; Ko et al., 2011). Además RFID se puede utilizar como solución a cuestiones de cambio de propiedad dentro del ciclo de vida de un producto individual (Kapoor et al., 2011). Por otra parte, se ha estudiado el proceso de adopción de RFID en la cadena de suministro con el objetivo de encontrar las características organizacionales clave ligadas a su éxito (Kim y Garrison, 2010; Nativi y Lee, 2012).

Manufactura: En el caso de la industria manufacturera, se cree que RFID puede ayudar a las empresas a rastrear activos y monitorear con mayor precisión los indicadores clave de desempeño (Ngai et al., 2012), hacer más visibles las operaciones de manufactura y permitir la toma de decisiones basada en información en tiempo real (Wang et al., 2010). Además, en el caso de externalización de tareas, RFID provee la capacidad de rastreo en tiempo real para la colaboración, el control y el monitoreo remoto entre los socios (Trappey et al., 2009). Otra aplicación en manufactura es la identificación de piezas para realizar operaciones de montaje robotizadas (Makris et al., 2012).

Minería: En lo concerniente a minería, se ha analizado principalmente la gestión de inventario de los detonadores, y la seguridad y el rastreo de explosivos y detonadores (Mishra et al., 2012). 
Retail: El valor de RFID para las operaciones de comercio Retail apunta en particular el uso de la tecnología para automatizar las decisiones de aprovisionamiento de estantes (Condea et al., 2012) y la reducción de la mala colocación de los productos (Rekik et al., 2008). Además, esta tecnología promete entregar muchos beneficios a los clientes de estos negocios, sin embargo, la aceptación por parte de estos aún es un tema controvertido (Müller-Seitz et al., 2009).

Servicios postales y de entrega: En el área de los servicios postales, las aplicaciones de RFID son principalmente la clasificación automática de correspondencia y paquetes, el rastreo de despachos y el control e inspección de buzones (Zhang et al., 2006).

Control de tráfico automovilístico: RFID también proporciona utilidad en el área automovilística para la recolección de información, el control de vehículos, y la identificación de vehículos robados o ilegales (Wen, 2010).

Turismo: El uso de RFID en el sector turismo se divide principalmente en cuatro categorías: el rastreo y control de humanos (turistas, usuarios, clientes), que va desde los pasaportes electrónicos hasta los sistemas de gestión de fidelidad de los clientes; sistemas de rastreo de activos; sistemas de pago sin contacto; y finalmente, dispositivos de información, que permiten a los usuarios obtener información de los objetos cercanos (Öztayşi et al., 2009).

Educación: En el área de educación, RFID puede ser utilizado para registrar la entrada de los alumnos, el control de acceso a las salas de clase o laboratorios, la gestión de atención en clases, sistemas de anuncios e información electrónica, administración de dinero electrónico, y automatización de los servicios bibliotecarios escolares (Akpınar y Kaptan, 2010).

\section{Áreas del conocimiento en ingeniería relacionadas con RFID}

En las carreras de Ingeniería Industrial podemos encontrar tres grandes áreas: Ciencias básicas, ciencias de las ingenierías y asignaturas de especialidad.

Ciencias Básicas: Si bien las asignaturas de ciencias básicas son consideradas por algunos como soporte del conocimiento que el futuro ingeniero cimentará en el transcurso de su instrucción, las nuevas corrientes basadas en enfoques de competencias, exigen que estas materias se contextualicen bajo un marco práctico, para su mayor entendimiento y correcta aplicación en su vida laboral, como lo son las asignaturas de Electromagnetismo (que puede incorporar la tecnología RFID, identificando los alcances con respecto a la radiofrecuencia, especialmente sus potencialidades y limitaciones técnicas), y la asignatura de Programación y Computación (con los lenguajes de computación aplicados y/o compatibles, así como estructuras de programación básica).

Ciencias de la Ingeniería: Son aquellas asignaturas pertenecientes a las ciencias transversales a las distintas especialidades en el ámbito de las ingenierías para el desempeño del futuro ingeniero. En asignaturas como Procesos de Fabricación e Investigación de Operaciones, se puede utilizar las bondades de la radiofrecuencia en cuanto a la trazabilidad para mejorar el control de sus procesos y aumentar su eficiencia. Asimismo en asignaturas como Ciencia de los Materiales, puede ser utilizada como receptor del historial de la información respecto a materiales de construcción utilizados en una obra. En asignaturas del área de la minería y en Prevención de Riesgos, se puede identificar como una herramienta de apoyo para la disminución de accidentes.

Asignaturas de Especialidad: En cuanto a las asignaturas de especialidad, la radiofrecuencia puede ser utilizada como parte de solución en problemáticas de un amplio espectro, participando en la concepción y diseño de sistemas de diferentes ámbitos en la línea profesional de Tecnologías \& Sistemas compuesta por asignaturas tales como Tecnologías de la Información, Ingeniería de Software, Teoría de Sistemas y Sistemas de Información \& Gestión. En el área de Producción puede ser utilizada como apoyo a los procesos estudiados, en asignaturas tales como Diseño y Planificación de la Producción, Procesos Industriales y Gestión de la Producción. En asignaturas como Gestión del Capital Humano, se puede ver la implicancias técnicas, operativas y éticas, con respecto a la utilización esta esta tecnología en el elemento humano, así como en la asignatura Derecho Empresarial, se pueden analizar los trasfondos legales de su utilización, especialmente en lo que respecta a la privacidad de las personas y sus acciones con respecto a un entorno organizativo. Finalmente, en asignaturas tales como Innovación \& Emprendimiento, pueden ser analizados nuevos alcances prácticos, utilizaciones, o nuevas ideas de negocio, que el mismo estudiante pudiera forjar para impactar a la sociedad con una solución que para el mercado tenga valor. 


\section{DISCUSIÓN}

Existiendo en las universidades un modelo educativo donde se expliciten la formación docente y el trabajo escolar con base en problemas, casos y proyectos, se puede desarrollar procesos innovadores para enseñar a aprender mediante estrategias que faciliten el desarrollo de competencias cognitivas de alto nivel (Muñoz-Cano et al., 2012). Duart (2006), indica que la estrategia es un arte que, al igual que otros, si bien acepta consejos estos son siempre de difícil aplicación directa. A pesar de ello, lo mejor en estos casos es aprender de lo que otros han realizado, de sus planificaciones y de sus resultados, e intentar trabajar en su adaptación a la realidad de cada institución. No existe, por tanto, una estrategia, sino diversas. No existen tampoco unas estrategias buenas y otras malas, todo depende, de la capacidad de entender al usuario, al estudiante, y de valorar adecuadamente sus necesidades de aprendizaje. La figura 2 muestra algunos elementos básicos a tener en cuenta en la tarea de elaboración de una estrategia de introducción de un proceso de innovación institucional. Se trata, a su vez, de un modelo de análisis, adaptado del modelo estratégico de Stonich.

Esquema de análisis estratégico

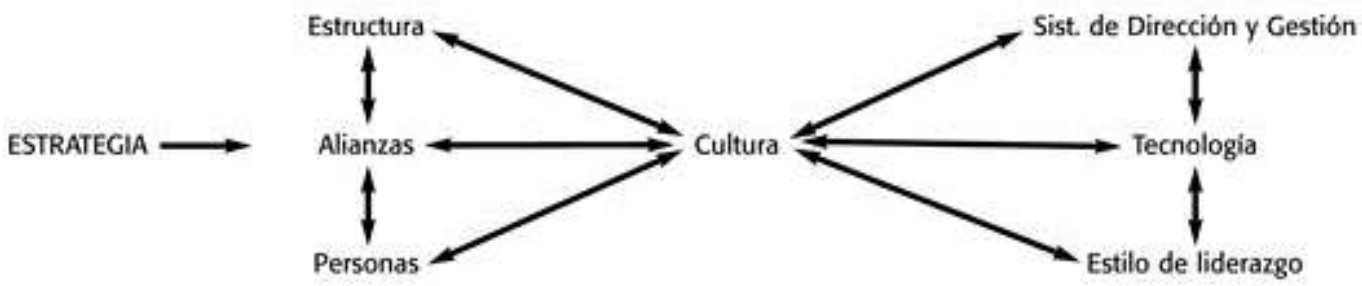

Fig. 2: Modelo de análisis estratégico para la introducción de una innovación (Duart, 2006)

\section{Estrategias}

Tomando en consideración las variables expuestas en el anterior modelo, y debido a que la adopción de una tecnología con un gran potencial de crecimiento, en diferentes ámbitos de acción tal como lo es la de radiofrecuencia, se sugieren las siguientes estrategias:

Alianzas con Proveedores de la Tecnología: para la obtención de los insumos de investigación y conocimientos técnicos por parte del productor, para lograr mayor eficiencia en la comprensión y utilización de la tecnología. Así mismo, los proveedores tendrán la retroalimentación necesaria sobre estándares requeridos, según las nuevas utilizaciones o de procesos de negocios identificados con gran crecimiento potencial.

Búsqueda de Partners Tecnológicos: con el propósito de adquirir conocimientos complementarios necesarios para la adopción de las nuevas tecnologías.

Ejecución de Proyectos de Vinculación con el Medio: La universidad, en alianza con empresas u organizaciones externas, podría realizar proyectos de cooperación mutua para implementar la tecnología RFID en procesos claves, que ayude a mejorar la competitividad regional en los sectores sensibles.

Creación de un Centro de Estudios: en alianza y/o con apoyo gubernamental, con el propósito de encauzar la utilización de la tecnología, dentro de los focos estratégicos requeridos según las directrices de estado que buscan potenciar áreas de la economía o de las industrias de relevancia.

Creación de un Centro de Negocios Tecnológicos: para vincularse con el empresariado para mostrar las bondades de la tecnología, así como para captar las necesidades in situ de los diversos usuarios de la tecnología, así como de potenciales clientes.

\section{La tecnología en el aula}

Así mismo, la adopción de la tecnología para la resolución de problemas en el aula, puede dar lugar que los estudiantes aborden situaciones que pueden resolverse según variados enfoques, propiciando de esta manera la estimulación del pensamiento creativo, el análisis crítico, el razonamiento y la iniciativa personal y comunitaria. Además, el desarrollo de trabajos grupales, favorecerían la formación en el campo de las relaciones interpersonales, en el trabajo cooperativo y en las competencias comunicativas de su especialidad (Castells et al., 2008). Esta tecnología, al igual que otros temas relacionados con la ingeniería, 
hace atractivo y necesario discutirlos en cursos universitarios, justificando su inclusión en cursos formales de ingeniería y ciencias, proponiendo formas de motivar e incentivar a los alumnos a través de trabajo de investigación, presentaciones orales e informes escritos (Valderrama et al., 2011).

Algunos ejemplos de cómo puede ser abordada esta tecnología a nivel universitario, se detallan a continuación:

Trabajos prácticos en el aula: como parte de trabajos semestrales, explicando la tecnología, o utilizándola como parte de aplicaciones en empresas u organizaciones, integrando la arquitectura de la solución.

Trabajos de Titulación: se pueden desarrollar trabajos de titulación para resolver las diferentes problemáticas empresariales u organizacionales, en los ámbitos descritos de las áreas de la ingeniería industrial, destacando la logística, control, trazabilidad, entre otras aplicaciones que se pueda requerir por las partes interesadas. Para el desarrollo de estos proyectos, se sugiere trabajar en un equipo multidisciplinario, para entregarle una robustez mayor a la solución propuesta. Además, esta tecnología puede ser utilizada como un sistema automático de recolección de datos para su posterior utilización y gestión, en sistemas con data Warehouse, Data Mining o Knowledge Discovery in Databases.

Investigación: si bien en los últimos años se ha avanzado en la aplicación de la tecnología en diferentes empresas, aún quedan dudas con respecto a la eficacia que ésta tendría, con respecto a las limitantes tecnológicas para algunos procesos en particular, como lo son el alcance de lectura, autonomía y capacidad de almacenar datos dinámicos. Las universidades, mediante equipos multidisciplinarios, podrían abordar estas interrogantes, para clarificar el campo de acción que puede tener el RFID, en distintos procesos de negocios con potencial de crecimiento.

\section{CONCLUSIONES}

Se concluye de que si bien, la tecnología RFID no es nueva, se ha visto potenciada con el desarrollo que han tenido otras tecnologías de la información, las cuales le han permitido una masificación y diversificación de los usos que podría llegar a tener. En este contexto las universidades en general, y las unidades que imparten carreras asociadas a la Ingeniería Industrial, tienen una gran oportunidad de potenciar su línea de acción al incorporar esta tecnología en la resolución de problemas de procesos empresariales y organizacionales, debido a que se encuentra alineada con las necesidades en las cuales se encuentran inmersas estas sociedades hoy en día. Así mismo, se les entregarían a los estudiantes, herramientas de alto impacto, para la realización de soluciones innovadoras, acordes a las exigencias del mundo de hoy.

\section{AGRADECIMIENTOS}

El estudio realizado fue financiado por la Dirección de Investigación de la Universidad de La Serena.

\section{REFERENCIAS}

Abad, E., Palacio, F., Nuin, M., Zárate, A.G.D., Juarros, A., Gómez, J.M. y Marco, S., RFID smart tag for traceability and cold chain monitoring of foods: Demonstration in an intercontinental fresh fish logistic chain, Journal of Food Engineering, 93(4), 394-399 (2009).

Akpinar, S. y Kaptan, H., Computer aided school administration system using RFID technology, Procedia Social and Behavioral Sciences, 2(2), 4392-4397(2010).

Aliaga, C., Ferreira, B., Hortal, M., Pancorbo, M.Á., López, J.M. y Navas, F.J., Influence of RFID tags on recyclability of plastic packaging, Waste Management, 31(6), 1133-1138 (2011).

Ampatzidis, Y.G. y Vougioukas, S.G., Field experiments for evaluating the incorporation of RFID and barcode registration and digital weighing technologies in manual fruit harvesting, Computers and Electronics in Agriculture, 66(2), 166-172 (2009).

Bendavid, Y. y Boeck, H., Using RFID to improve hospital supply chain management for high value and consignment items, Procedia Computer Science, 5, 849-856 (2011).

Björk, A., Erlandsson, M., Häkli, J., Jaakkola, K., Nilsson, Å., Nummila, K. y Sirkka, A., Monitoring environmental performance of the forestry supply chain using RFID, Computers in Industry, 62(8), 830-841 (2011).

Bottani, E., Montanari, R. y Volpi, A., The impact of RFID and EPC network on the bullwhip effect in the Italian FMCG supply chain, International Journal of Production Economics, 124(2), 426-432 (2010). 
Çakici, Ö.E., Groenevelt, H. y Seidmann, A., Using RFID for the management of pharmaceutical inventory system optimization and shrinkage control, Decision Support Systems, 51(4), 842-852 (2011).

Castells, M., Arese, A., Albizzati, E. y Rossetti, G.H., Propuesta para la Enseñanza de la Ingeniería: Un Espacio Curricular Creado desde la Investigación-Acción, Formación Universitaria, 1(2), 9-16 (2008).

Chae, S. y Yoshida, T., Application of RFID technology to prevention of collision accident with heavy equipment, Automation in Construction, 19(3), 368-374 (2010).

Condea, C., Thiesse, F. y Fleisch, E., RFID-enabled shelf replenishment with backroom monitoring in retail stores, Decision Support Systems, 52(4), 839-849 (2012).

Costin, A., Pradhananga, N. y Teizer, J., Leveraging passive RFID technology for construction resource field mobility and status monitoring in a high-rise renovation project, Automation in Construction, 24, 1-15 (2012).

Domdouzis, K., Kumar, B. y Anumba, C., Radio-Frequency Identification (RFID) applications: A brief introduction, Advanced Engineering Informatics, 21(4), 350-355 (2007).

Duart, J.M., Estrategias en la introducción y uso del e.Learning en educación superior, Educación médica, 9(2), 13-20 (2006).

Fisher, J.A. y Monahan, T., Tracking the social dimensions of RFID systems in hospitals, International Journal of Medical Informatics, 77(3), 176-183 (2008).

Hohberger, C., Davis, R., Briggs, L., Gutierrez, A. y Veeramani, D., Applying radio-frequency identification (RFID) technology in transfusion medicine, Biologicals, 40(3), 209-213 (2012).

Hong, I.H., Dang, J.F., Tsai, Y.H., Liu, C.S., Lee, W.T., Wang, M.L. y Chen, P.C., An RFID application in the food supply chain: A case study of convenience stores in Taiwan, Journal of Food Engineering, 106(2), 119126 (2011).

IDTECHEX, Global RFID market will reach $\$ 7.88$ billion in 2013 (2013), http://www.idtechex.com/research/articles/global-rfid-market-will-reach-7-88-billion-in-2013-00005914.asp.

Acceso: 10 de Febrero (2014).

Jedermann, R., Ruiz-Garcia, L. y Lang, W., Spatial temperature profiling by semi-passive RFID loggers for perishable food transportation, Computers and Electronics in Agriculture, 65(2), 145-154 (2009).

Kapoor, G., Zhou, W., y Piramuthu, S., Multi-tag and multi-owner RFID ownership transfer in supply chains, Decision Support Systems, 52(1), 258-270 (2011).

Kim, S., y Garrison, G., Understanding users' behaviors regarding supply chain technology: Determinants impacting the adoption and implementation of RFID technology in South Korea, International Journal of Information Management, 30(5), 388-398 (2010).

Knospe, H., y Pohl, H., RFID security, Information Security Technical Report, 9(4), $39-50$ (2004).

Ko, J.M., Kwak, C., Cho, Y. y Kim, C.O., Adaptive product tracking in RFID-enabled large-scale supply chain, Expert Systems with Applications, 38(3), 1583-1590 (2011).

Kranzfelder, M., Zywitza, D., Jell, T., Schneider, A., Gillen, S., Friess, H., y Feussner, H., Real-Time Monitoring for Detection of Retained Surgical Sponges and Team Motion in the Surgical Operation Room Using Radio-Frequency-Identification (RFID) Technology: A Preclinical Evaluation, Journal of Surgical Research, 175(2), 191-198 (2012).

Laniel, M., y Émond, J.P., Mapping of RFID tag readability in relation to the food content in a refrigerated sea container at 915\&\#xa0;MHz, Innovative Food Science \& Emerging Technologies, 11(4), 703-706 (2010).

Lee, C.K.M., Ho, W., Ho, G.T.S., y Lau, H.C.W., Design and development of logistics workflow systems for demand management with RFID, Expert Systems with Applications, 38(5), 5428-5437 (2011).

Leimeister, S., Leimeister, J. M., Knebel, U., y Krcmar, H., A cross-national comparison of perceived strategic importance of RFID for CIOs in Germany and Italy, International Journal of Information Management, 29(1), 37-47 (2009).

Liao, W.P., Lin, T.M.Y., y Liao, S.H., Contributions to Radio Frequency Identification (RFID) research: An assessment of SCI-, SSCl-indexed papers from 2004 to 2008, Decision Support Systems, 50(2), 548-556 (2011).

Lu, W., Huang, G.Q. y Li, H., Scenarios for applying RFID technology in construction project management, Automation in Construction, 20(2), 101-106 (2011). 
Majrouhi Sardroud, J., Influence of RFID technology on automated management of construction materials and components, Scientia Iranica, 19(3), 381-392 (2012).

Makris, S., Michalos, G. y Chryssolouris, G., RFID driven robotic assembly for random mix manufacturing, Robotics and Computer-Integrated Manufacturing, 28(3), 359-365(2012).

Mishra, P.K., Bolic, M., Yagoub, M.C.E. y Stewart, R.F., RFID technology for tracking and tracing explosives and detonators in mining services applications, Journal of Applied Geophysics, 76(0), 33-43 (2012).

Müller-Seitz, G., Dautzenberg, K., Creusen, U. y Stromereder, C., Customer acceptance of RFID technology: Evidence from the German electronic retail sector, Journal of Retailing and Consumer Services, 16(1), 31-39 (2009).

Munoz-Cano, J.M, Cordova, J.A. y Priego, H., Dificultades y facilidades para el desarrollo de un proceso de innovación educativa con base en las Tecnologías de la Información y Comunicación (TIC), Formación Universitaria, 5(1), 3-12 (2012).

Najera, P., Lopez, J. y Roman, R., Real-time location and inpatient care systems based on passive RFID, Journal of Network and Computer Applications, 34(3), 980-989 (2011).

Nativi, J. y Lee, S., Impact of RFID information-sharing strategies on a decentralized supply chain with reverse logistics operations, International Journal of Production Economics, 136(2), 366-377 (2012).

Ngai, E.W.T., Chau, D.C.K., Poon, J.K.L., Chan, A.Y.M., Chan, B.C.M. y Wu, W.W.S., Implementing an RFID-based manufacturing process management system: Lessons learned and success factors, Journal of Engineering and Technology Management, 29(1), 112-130 (2012).

Ngai, E.W.T., Moon, K.K.L., Riggins, F.J. y Yi, C.Y., RFID research: An academic literature review (19952005) and future research directions, International Journal of Production Economics, 112(2), 510-520 (2008a).

Ngai, E.W.T., Suk, F.F.C., y Lo, S.Y.Y., Development of an RFID-based sushi management system: The case of a conveyor-belt sushi restaurant, International Journal of Production Economics, 112(2), 630-645 (2008b).

Öztayşi, B., Baysan, S. y Akpinar, F., Radio frequency identification (RFID) in hospitality, Technovation, 29(9), 618-624 (2009).

Oztekin, A., Pajouh, F.M., Delen, D. y Swim, L.K., An RFID network design methodology for asset tracking in healthcare, Decision Support Systems, 49(1), 100-109 (2010).

Parlak, S., Sarcevic, A., Marsic, I. y Burd, R.S., Introducing RFID technology in dynamic and time-critical medical settings: Requirements and challenges, Journal of Biomedical Informatics, 45(5), 958-974 (2012).

Peris-Lopez, P., Orfila, A., Mitrokotsa, A. y Van Der Lubbe, J.C.A., A comprehensive RFID solution to enhance inpatient medication safety, International Journal of Medical Informatics, 80(1), 13-24 (2011).

Pirrone, J. y Huerta, M.K., RFID en el sector salud: aplicaciones, beneficios e incertidumbres, Universidad, Ciencia y Tecnología, 15(60), 144-150 (2011).

Qu, X., Simpson, L.T. y Stanfield, P., A model for quantifying the value of RFID-enabled equipment tracking in hospitals, Advanced Engineering Informatics, 25(1), 23-31 (2011).

Reiners, K., Hegger, A., Hessel, E.F., Böck, S., Wendl, G. y Van Den Weghe, H.F.A., Application of RFID technology using passive HF transponders for the individual identification of weaned piglets at the feed trough, Computers and Electronics in Agriculture, 68(2), 178-184 (2009).

Rekik, Y., Sahin, E., y Dallery, Y., Analysis of the impact of the RFID technology on reducing product misplacement errors at retail stores, International Journal of Production Economics, 112(1), 264-278 (2008).

Reyes, P.M., Li, S. y Visich, J.K., Accessing antecedents and outcomes of RFID implementation in health care, International Journal of Production Economics, 136(1), 137-150 (2012).

Roberts, C.M., Radio frequency identification (RFID), Computers \& Security, 25(1), 18-26 (2006).

Robshaw, M.J.B., An overview of RFID tags and new cryptographic developments, Information Security Technical Report, 11(2), 82-88 (2006).

Roh, J.J., Kunnathur, A. y Tarafdar, M., Classification of RFID adoption: An expected benefits approach, Information \& Management, 46(6), 357-363 (2009).

Ruiz-Garcia, L. y Lunadei, L., The role of RFID in agriculture: Applications, limitations and challenges, Computers and Electronics in Agriculture, 79(1), $42-50$ (2011). 
Sarac, A., Absi, N. y Dauzère-Pérès, S., A literature review on the impact of RFID technologies on supply chain management, International Journal of Production Economics, 128(1), 77-95 (2010).

Stankovskil, S., Ostojic, G., Senk, I., Rakic-Skokovic, M., Trivunovic., S. y Kucevic D., Dairy cow monitoring by RFID, Scientia Agricola, 69(1), 75-80 (2012).

Tajima, M., Strategic value of RFID in supply chain management, Journal of Purchasing and Supply Management, 13(4), 261-273 (2007).

Tan, T.H. y Chang, C.S, Development and evaluation of an RFID-based e-restaurant system for customercentric service, Expert Systems with Applications, 37(9), 6482-6492 (2010).

Trappey, A.J.C., Lu, T.H. y Fu, L.D., Development of an intelligent agent system for collaborative mold production with RFID technology, Robotics and Computer-Integrated Manufacturing, 25(1), 42-56 (2009).

Tzeng, C.T., Chiang, Y.C., Chiang, C.M. y Lai, C.M, Combination of radio frequency identification (RFID) and field verification tests of interior decorating materials, Automation in Construction, 18(1), 16-23 (2008).

Valderrama, J.O; Espindola, C. y Quezada, R., Huella de Carbono, un Concepto que no puede estar Ausente en Cursos de Ingeniería y Ciencias, Formación Universitaria, 4(3), 3-12 (2011).

Véronneau, S. y Roy, J., RFID benefits, costs, and possibilities: The economical analysis of RFID deployment in a cruise corporation global service supply chain, International Journal of Production Economics, 122(2), 692-702 (2009).

Wang, L.C., Enhancing construction quality inspection and management using RFID technology, Automation in Construction, 17(4), 467-479 (2008).

Wang, Y.M., Wang, Y.S. y Yang, Y.F., Understanding the determinants of RFID adoption in the manufacturing industry, Technological Forecasting and Social Change, 77(5), 803-815 (2010).

Wen, W., An intelligent traffic management expert system with RFID technology, Expert Systems with Applications, 37(4), 3024-3035 (2010).

Wu, N.C., Nystrom, M.A., Lin, T.R. y Yu, H.C., Challenges to global RFID adoption, Technovation, 26(12), 1317-1323 (2006).

Xianyan, W. y Jiayuan, L., The Applied Research of RFID Technology in Library Management, Energy Procedia (13), 7030-7036 (2011).

Zhang, M. y Li, P., RFID Application Strategy in Agri-Food Supply Chain Based on Safety and Benefit Analysis, Physics Procedia, 25(0), 636-642 (2012).

Zhang, X.D., Yue, S.J. y Wang, W.M., The review of RFID applications in global postal and courier services, The Journal of China Universities of Posts and Telecommunications, 13(4), 106-110 (2006).

Zhu, X., Mukhopadhyay, S.K. y Kurata, H., A review of RFID technology and its managerial applications in different industries, Journal of Engineering and Technology Management, 29(1), 152-167 (2012). 
